\title{
23.3: Tactile Sensor Based on Piezoelectric Resonance
}

\author{
G.Murali Krishna and K.Rajanna * \\ Department of Instrumentation \\ INDIANINSTITUTE OF SCIENCE \\ Bangalore, India. \\ * Corresponding author ,E-mail:kraj@isu.iisc.ernet.in
}

\begin{abstract}
We discuss here about the realization of tactile sensor based on the principle of change in piezoelectric resonancefrequency with the appliedpressure. An array of electrodes has been adopted on either side of the PZT material to have independent resonators. The common areas sandwiched between the electrodes and excitable at resonance frequency of the PZT material are used to form the sensitive area of the tactile sensor. The electrodes were deposited using sputtering technique. Tactile sensors with $3 \times 3,7 \times 7$ and $15 \times 15$ array of electrodes are developed with different electrode dimensions and separation between the electrodes. The tactile sensor has been interfaced to computerfor the convenience of automatic scanning and making it more user interactive. The tactile sensors developed with different spatial resolution were tested for different shaped objectsplaced in contact with the sensor. The $3 \times 3$ matrix tactile sensor showed relatively poor spatial resolution whereas 15x15-matrix tactile sensor showed improved spatial resolution. The sensor with $7 \times 7$ matrix elements was testedfor its sensitivity to different extents of applied force/pressure. The output response study carried out on the sensors indicated that these sensors can provide information not only about the extent of force/pressure applied on the object, but also the contour of the object which is in contact with the sensor.
\end{abstract}

\section{Keywords}

Tactile sensor, Piezoelectric Resonance and Robotics.

\section{INTRODUCTION}

Touch and tactile sensors are devices which measure the parameters of contact between the sensor and an object. Touch sensing is basically the process of detection and measurement of a contact force at a defined point. A touch sensor can also be restricted to binary information, namely touch and no touch. Whereas, tactile sensing is the process of detection and measurement of the spatial distribution of forces perpendicular to a predetermined sensory area, and the subsequent interpretation of the spatial information. A tactile sensing array can be considered to be a co-ordinated group of touch sensors [5]. Robotics and the industrial automation are the potential application areas which have generated considerable interest, because the contact interaction is the most fundamental feature of any physical system. However, there are many other potential application areas including agriculture, food processing, bio-medical [16, 10], entertainment, and future domestic and service industries. Different types of principles have been explored in realizing the tactile sensors. They are based on Mechanical [12, 6, 14], Resistive [17, 8], Capacitive [4], Magneto resistive [7], fiber optic [7], Piezoelectric [1, 11] and Surface Acoustic wave (SAW) [15] techniques. In our present work, we have used the principle of change in the piezoelectric resonance frequency of poled ceramic lead zirconium titanate (PZT) with the applied stress. We have adopted an array of electrodes to have independent resonators. The tactile sensor developed is interfaced to computer and automated to test its output behavior. The response of the tactile sensor has been tested for different shaped of objects placed in contact with the sensor. The sensor is also tested for different extent of pressures applied over the object placed in contact with the sensor.

\section{PRINCIPLE, DESIGN \& PREPARATION}

\section{A. Principle}

When an applied frequency to a piezoelectric material is equal to its mechanical resonance frequency, then the material is said to be in piezoelectric resonance. Some oscillator circuits when suitably connected with piezoelectric material will give output frequency at the mechanical resonance frequency of the material. The resonance frequency of the resonating material is given by equation 1 [2]

$$
f_{0}=\frac{1}{2 t} \sqrt{\frac{K}{\rho}}
$$

Where,

$t=$ Thickness of the piezoelectric material.

$f_{0}=$ Natural mechanical resonance frequency of first order.

$\mathrm{K}=$ Stiffness constant of the material.

$\rho=$ Density of the material.

The area between the electrodes that is oscillating at the mechanical resonance frequency of the piezoelectric 
material is the active area. For a given material, if the stiffness constant and density of the material are kept constant, then the frequency becomes a sensitive parameter of thickness. When force/pressure is applied on the active area, strain on the active area occurs and the resonance frequency increases [3]. The change in resonance frequency A is given by [13]

$$
\frac{\Delta f}{f_{0}}=\frac{f-f_{0}}{f_{0}}=A F
$$

Where,

$f=$ Resonance frequency after the application of pressure.

A $f=$ Change in the resonance frequency.

$\mathrm{A}=$ Constant.

$\mathrm{F}=$ Applied force.

If stiffness constant $\mathrm{K}$ and density $\rho$ of a given material are constant, then from equation(1),

$$
f \alpha \frac{1}{\bar{t}}
$$

Hence,

$$
\frac{\Delta f}{f_{0}}=-\frac{\Delta t}{t}=A F
$$

where, At is thickness deformation. The negative sign implies that, when force/pressure is applied on the resonating area, thickness decreases and hence the frequency increases. When $\mathrm{A} f$ is far less than $f_{0}$, then [13]

$$
\Delta f \alpha F
$$

The change in frequency is directly proportional to the applied forcelpressure.Using this basic principle an array type tactile sensor is realized in the present work.

\section{Design and Preparation}

We have used the commercially available circularly shaped poled PZT material for realizing the tactile sensor. The PZT material used is of thickness $1 \mathrm{~mm}$ and diameter $20 \mathrm{~mm}$ with a resonance frequency $-1 \mathrm{MHz}$. Initially, the linear variation of change in resonance frequency with applied pressure/force for the chosen PZT samples was ascertained. Further, an array of electrodes are deposited on either side of the PZT material such that the array of electrodes on one side are perpendicular to the array of electrodes on the other side. DC magnetron sputtering technique has been used for depositing the array of electrodes. In order to obtain the required array pattern of electrodes, precision mechanical masks were employed during deposition. These masks were designed using AUTOCAD and prepared by adopting chemical milling technique. Double enameled copper wires were attached to the electrodes using temperature controlled soldering station. The common areas of PZT material sandwiched between the intersecting rejoins of the electrodes form a matrix of independently excitable resonators at the resonance frequency of the material (figure 1). By selecting one electrode from the top and one from the bottom, the common area between the electrodes is excitable at resonance. This procedure can be repeated for all the matrix of common areas between the electrodes. Each time, the matrix location and its corresponding resonance frequency need to be noted. This becomes the reference data of the sensor before detecting any object. In the present work, the tactile sensors with $3 \times 3,7 \times 7$ and $15 \times 15$ array of electrodes have been prepared. In each case, the width of array electrodes and the separation between them were suitably chosen [9].

\section{TESTING OF THE TACTILE SENSOR}

In order to study the out put response of the tactile sensor, the object to be studied was placed in contact with the sensor and subjected to different extent of pressures/force using an arrangement [9] consisting of a micrometer with digital display and capable of measuring the displacement/deformation up to $+/$ - 1micron accuracy. Small pieces of silicon rubber covering the electrodes region were placed on either side of the sensor. These silicon pieces ensure smooth transfer of the applied force and avoid the possible shorting (especially in case of conducting objects) between the electrode arrays. In the present study, different size and shaped objects were used. Keeping the object in contact with the sensor, the entire matrix of elements are excited at resonance one after the other and their positions with corresponding frequencies are recorded. The matrix of elements subjected to pressure show an increase in frequency proportional to the extent of pressure applied. The difference in frequency (before keeping the object and after keeping the object) is calculated. It is important to note that, wherever the object is in contact with the sensor, the corresponding elements in the matrix only show an increase in the frequency. In order to obtain a contour of the object that is in contact with the sensitive area of the tactile sensor, a 3D plot was made between the position of the matrix elements in the $X-Y$ axes plane and the change in frequency on the $\mathrm{Z}$-axis. The process of doing the above operation manually by selecting the individual matrix elements is time consuming and tedious. Therefore, in order to make the whole process automatic and more user interactive, necessary electronic circuits and software programs were designed and implemented to interface the sensor with the computer [9]. 


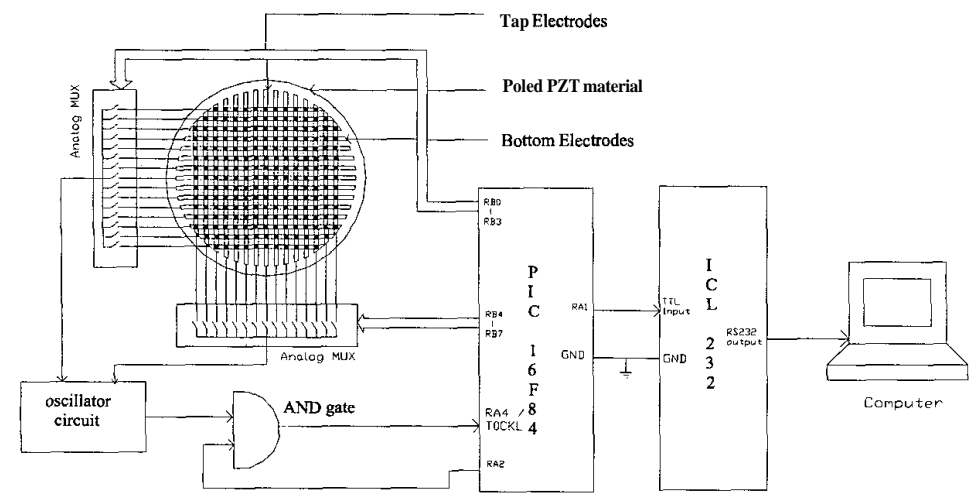

Figure 1: A block diagram of the experimental arrangement for testing the out put response of the tactile sensor.

A block diagram of the complete experimental arrangement along with the tactile sensor is shown in figure 1. It consists of analog multiplexers, oscillator ciruit, micro controller and a level converter for RS 232 interface to the computer. The program written in the micro -controller initially selects a matrix element and the selected matrix element gets connected to the oscillator circuit. The frequency counter program will measure the oscillator output frequency and sends the measured frequency to computer through RS 232 level converter. This procedure gets repeated till the complete scanning of entire matrix of elements. Hence, the entire operation of scanning the matrix of active elements, measuring the frequency and sending the measured frequency to computer is controlled by the micro-controller. All the tactile sensors made have been tested for their out put response using the above experimental arrangement and procedure.

\section{RESULTS AND DISCUSSION}

The tactile sensors with different number of arrays of electrodes namely $3 \times 3$ arrays, $7 \times 7$ arrays and $15 \times 15$ arrays were studied for their out put response by using the aluminum plate (both square and circular shaped) as an object placed over the sensor. In all the figures shown below, left side of the figures signifies the size of the sensitive area, with hashed region showing the location of the object over this sensitive area and the right side of the figures shows the corresponding response. Figure 2 shows the response for the object covering the entire sensitive area of the $3 \times 3$ array tactile sensor. The response corresponding to regions between the active elements is linearly interpolated from the data obtained from the active elements of the sensor. As can be seen in figure 2, the response is higher at the center compared to the regions towards the edges.
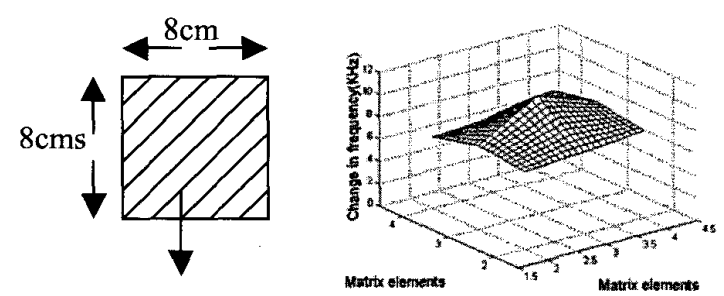

Location of object

Figure2: Response of $3 \times 3$ array tactile sensor for the object covering the entire active region.

This is thought to be due to several possible reasons like non-uniformity of applied pressure, non-uniform poling of the PZT material or could be due to non-uniformity in thickness of the PZT ceramic material used. The ambiguity in non-uniformity in applied pressure can be resolved by testing the sensor with higher spatial resolution (more number of matrix elements).
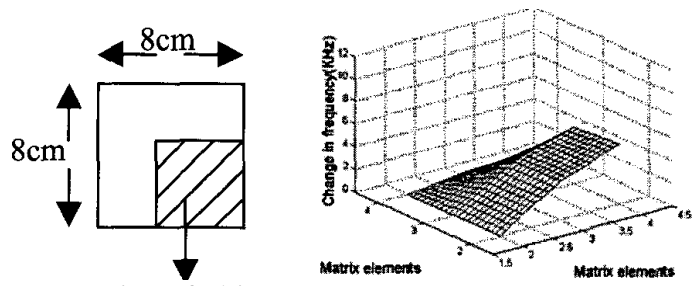

Location of object

Figure 3: Response of 3×3 array tactile sensor for the object located at one corner side of the active area.

The response of the $3 \times 3$ array sensor for the object located towards one side of the active area is shown in figure 3 . It is clearly evident in this case, that the response of the sensor is largely confined to the area covered by the object. 

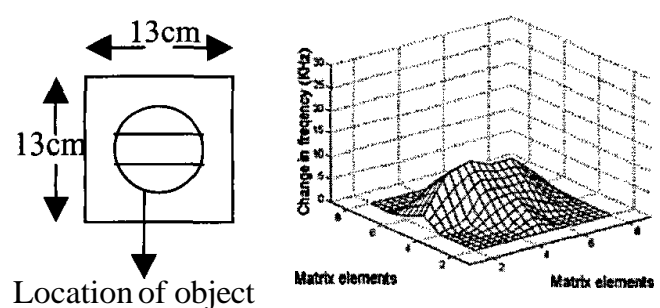

Figure 4: Response of $7 \times 7$ array tactile sensor for a circular object located at the center of the active area.

The response of the $7 \times 7$ array tactile sensor for a circular object located at the centre of the active area is shown in figure 4. It can be observed that, the response of the sensor is not only confined to the actual area of the object which is in contact with the sensor, but also to the adjacent regions beyond the edge boundaries of the object. This is attributed to the fact that, when pressure is applied over the object, it is transferred to the active area through the silicon rubber.

Since the silicon rubber is elastic, it expands under the application of pressure and cover the adjacent additional regions of the active area of the sensor.
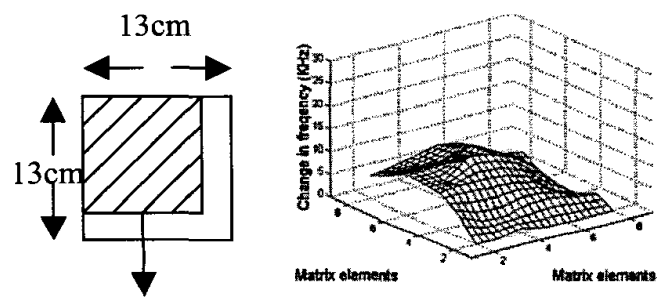

Location of object

Figure 5: Response of $7 \times 7$ array tactile sensor for a square shaped object located towards one corner ofthe active area
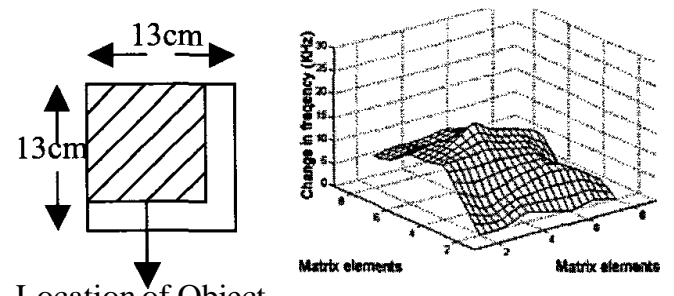

Location of Object

Figure 6: Response of the $7 \times 7$ array tactile sensor for a square shaped object located towards one corner side of the active area (same as in the case of figure 5) and with increased extent of pressure/force.
The response of the $7 \times 7$ array tactile sensor in case of the square shaped object located towards one comer side of the active area is shown in figure 5. The response of the sensor for the same object located at the same position but with the increased extent of pressure is shown in figure 6 . The change in frequency is more for the higher extent of applied pressure indicating the pressure sensitivity of the sensor. Also, it is clearly evident that, in comparision with the $3 \times 3$ array sensor, the spatial resolution of $7 \times 7$ array tactile sensor has improved significantly as expected.

The response of the tactile sensor with $15 \times 15$ array of electrodes is shown in figure 7 for a circular object placed at the centre of the active area. Also, the response of the same sensor for an object having a hole at the centre is shown in figure 8.

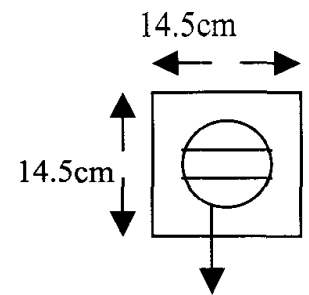

Location of object

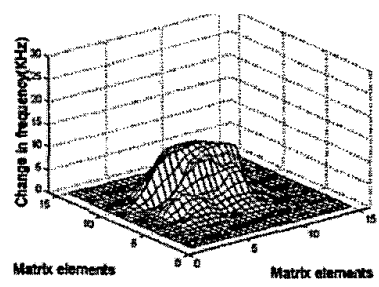

Figure 7: Response of the $15 \times 15$ array tactile sensor for a circular object placed at the center of the active area.
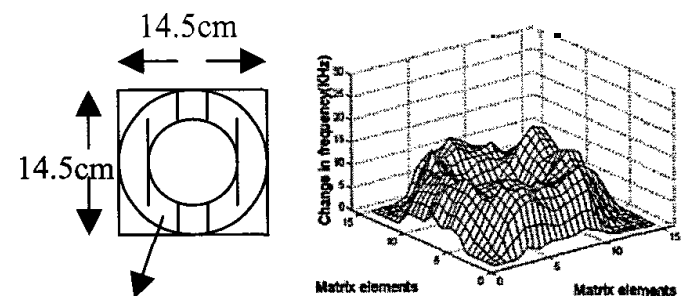

Location'of object

Figure 8: Response of the $15 \times 15$ array tactile sensor for an object having a hole at the center.

It is clearly noticeable that, compared to $7 \times 7$ array sensor, the spatial resolution has further improved. It is also evident from figures $7 \& \mathbf{8}$ that, the sensor can provide information about contour of the object. 


\section{Conclusions}

Successfully developed the tactile sensor based on the principle of piezoelectric resonance . An array of electrodes geometry has been employed in the realization of the sensor. The necessary electronics (both hardware and software) has been developed and the entire assembly is interfaced suitably with the computer. The out put response study carried out on the sensor clearly demonstrate that the sensor can provide information not only about the extent of force /pressure applied on the object, but also the contour of the object which is in contact with the sensor. Scope exists for improving the sensor behavior in terms of its spatial resolution by increasing the number of array electrodes using lithographic techniques. Presently developed tactile sensor has the potential application possibilities in the areas of robotics and biomedical engineering.

\section{Acknowledgement}

Authors gratefully thank Chairman, Department of Instrumentation, IISc for his kind support and encouragement in carrying out this work. Heartfelt thanks are due to Dr. M.M.Nayak, LPSC, ISRO for his kind help in many ways and the fruitful discussions related to this work. Thanks are also due to Dr.L...Shivalingappa for his help in the early stages of this work.

\section{References:}

[1] Barsky,M.F., Lindner,D.K. and Claus,R.O., Robot Gripper Control system using PVDF piezoelectric sensors, IEEE Trans. on Ultrasonics, Ferroelectrics and Frequency Control, Vol 36, No.1, pp 129-133, Jan 1989.

[2] Benes,E., Groschl,M., Burger,W. and Schmid,M., Sensors based on piezoelectric resonators, Sensors and Actuators - A 48, pp 1-21, 1995.

[3] Gehin,C., Barthod,C. and Teisseyre,Y., Design and characterization of new force resonant sensor, Sensors and Actuators - A 84, pp 65-69,2000.

[4] Hiroki Morimura, Satoshi Shigematsu and Katsuyuki Machinda, A novel sensor cell architecture and sensing circuit scheme for capacitive fingerprint sensors, IEEE Journal of Solid-state Circuits, Vol - 35, pp 724-731 2000.

[5]http://www.soton.ac.uk/ rmc1/robotics/artactile.htm

[6] Jayawant,B.V., Onorio,M.A. \& MacWatson,J.D., Robot Tactile sensing: A new array sensor, Robot Sensors II, Tactile and Non-vision, Edited by A.Pugh, IFS, London, pp 199-205,1986.
[7] Kazuo Tanie, Advances in Tactile sensors for robotics, Proceedings of the 6th sensor symposium, Japan, pp 63-68, 1986.

[8] Litian Liu, Xinyu Zheng and Zhijian Li, An array tactile sensor with piezoresistive single crystal silicon diaphragm, Sensors and Actuators -A32,pp193-196,1993.

[9] Murali Krishna,G., Development of tactile sensors based on piezoelectric resonance and surface acoustic wave techniques, M.Sc(Engg.) Thesis, 2001, Indian Institute of Science, Bangalore, India.

[10] Neumann,M.R., Medical progress through technology, Vol 9, Springer, New York, pp 951982.

[11] Omata,S. and Terunuma,Y., New tactile sensor like human hand and its applications, Sensors and Actuators A, Vol. 35, pp $9-15$

1992.

[12] Pagh,P., Heginbotham,W.B. and Page,C.J., Novel techniques for tactile sensing in a three dimensional environment, Ind. Robot. Mar, pp 35-48,1977.

[13] Ping Li and Yurnei Wen, An arbitrarily distributed tactile piezoelectric sensor array, Sensors and Actuators A 65, pp 141-146, 1998.

[14] Rebman,J. and Morris,K.A., A tactile sensor with Electro optic transduction, Proc. 3rd Int. Conf. Robot Vision and Sensory controls, Cambridge, MA, USA, Nov, pp2 10-216,1982.

[15] Robert Adler and Peter J. Desmares, An economical touch panel using SAW absorption, IEEE Transactions on Ultrasonics, ferroelectrics and frequency control, Vol UFFC - 34, pp 195-201,1987.

[16] Sorab,J., Allen,R.H. and Gonik,B., Tactile sensory monitoring of clinician-applied forces during delivery of new borns, IEEE Transactions on Bio-medical Engineering, Vol 35, pp.1090-1093,December,1998.

[17] Susumu Sugiyama, Ken Kawahata, Masakazu Yonendu and Isemi Igarashi, Tactile image detection using $1 \mathrm{~K}$ - element silicon pressure sensor array, Sensors and Actuators - A21-A23, pp 397-400, 1990. 